\title{
The Impact of Visual Feedback on the Motor Control of the Upper-Limb
}

\author{
O. Urra, A Casals, Senior Member, IEEE, and R, Jané, Senior Member, IEEE
}

\begin{abstract}
Stroke is a leading cause of adult disability with upper-limb hemiparesis being one of the most frequent consequences. Given that stroke only affects the paretic arm's control structure (the set of synergies and activation vectors needed to perform a movement), we propose that the control structure of the non-affected arm can serve as a physiological reference to rehabilitate the paretic arm. However, it is unclear how rehabilitation can effectively tune the control structure of a patient. The use of Visual Feedback (VF) is recommended to boost stroke rehabilitation, as it is able to positively modify neural mechanisms and improve motor performance. Thus, in this study we investigate whether VF can effectively modify the control structure of the upper-limb. We asked six neurologically intact subjects to perform a complete upper-limb rehabilitation routine comprised of 12 movements in absence and presence of VF. Our results indicate that VF significantly increases interlimb similarity both in terms of synergies and activation coefficients. However, the magnitude of improvement depended upon each subject. In general, VF brings the control structure of the nondominant side closer to the control structure of dominant side, suggesting that VF modifies the control structure towards more optimized motor patterns. This is especially interesting because stroke mainly affects the activation coefficients of patients and because it has been shown that the control of the affected side resembles that of the nondominant side. In conclusion, VF may enhance motor performance by effectively tuning the control-structure. Notably, this finding offers new insights to design improved stroke rehabilitation.
\end{abstract}

\section{INTRODUCTION}

Stroke is the main cause of serious long-term disability, due to the relative ineffectiveness of current rehabilitation strategies [1]. Upper-limb hemiparesis is one of the most common post-stroke consequences. Therefore, improving upper-limb rehabilitation is a key aspect to boost the quality of life of patients with stroke, and reduce the huge sociosanitary costs associated.

* This work was supported by grants from La Caixa Foundation and Secretaria d'Universitats $i$ Recerca del Departament d'Economia $i$ Coneixement de la Generalitat de Catalunya (Consolidated research group GRC: 2014 SGR 1569), The work was conducted under the framework of the HYPER project (CSD2009-00067 CONSOLIDER INGENIO 2010).

O. Urra, A. Casals and R. Jané are with the Universitat Politècnica de Catalunya - BarcelonaTech and Institute for Bioengineering of Catalonia (IBEC), Baldiri Reixac 4, Tower I, $9^{\text {th }}$ floor, 08028, Barcelona, Spain (phone: +34 (93) 4039706; fax: +34 (93) 4039702; e-mail: ourra@ibecbarcelona.eu, acasals@ibecbarcelona.eu, rjane@ ibecbarcelona.eu) R. Jané is also with CIBER de Bioingeniería, Biomateriales y Nanomedicina, Spain.
Our brain achieves motor coordination by combining simultaneous activations of diverse muscle groups known as synergies [2-3]. In this framework, a control structure is defined as the set of synergies and activation coefficients needed to perform a movement. Recent studies demonstrate that stroke may affect the motor capabilities of a patient by altering the control structure of the paretic side $[4,5]$. Yet, the control structure of the non-affected side remains apparently intact [6]. Thus, we propose using the control structure of the non-affected side as a reference to rehabilitate the paretic arm. In a previous study we provided sound evidence in favor of this hypothesis, by demonstrating that the inter-limb similarity of the control structure is substantially higher than that found across subjects [7]. However, it is still not clear the way in which rehabilitation can effectively modify the control structure.

Mirror Visual Feedback (VF) is one of the recommended strategies to enforce stroke rehabilitation [8], as it is simple, inexpensive and has shown promising results in the rehabilitation of stroke $[9,10]$. Although a number of authors have investigated the neurological effects of VF [11, 12], to our best knowledge the impact of VF in the control structure has not been addressed yet. In a preliminary study conducted on two simple upper-limb movements, we demonstrated that VF enhances the inter-limb similarity of the control structure [13]. Here, we have extended such study to the analysis of a complete upper-limb rehabilitation routine comprised of 12 movements. Participants repeated each routine with and without VF. EMG signals were collected from both arms and corresponding control structures were extracted. We evaluated the effect of VF by comparing the improvement in the inter-limb similarity of the control structures found between the limbs of the same subject when VF was present.

\section{MethodS}

\section{A. Experimental Protocol}

Six right-handed subjects (age 25-35) with no known neurological impairments participated in this study. The Institutional Review Board of the Institute for Bioengineering of Catalonia approved all the procedures used. Written informed consent was required for participation in the study. During the experimental session subjects had to perform a set of 12 exercises taken from standard upper-limb rehabilitation routines that covered the basic range of motion of elbow and shoulder. Each exercise consisted on a simple movement involving one-degree of freedom in elbow or shoulder. Subjects were asked to perform 30 repetitions of each 
movement with the two arms separately. Each set of 30 repetitions was done in absence and presence of VF. VF was provided by allowing the subjects to track their movements using a mirror that was placed orthogonal to the axis around which the movement was produced. Movement onset was indicated by auditory cues. One minute was allowed between each set of repetitions to rest and switch movement. In order to avoid learning effects, the arm order was randomly chosen for each subject.

At the end of the experimental session, all the subjects were asked to fill in a questionnaire to evaluate the overall experience and the sensations they perceived during the experiment. The questionnaire was comprised of 5 questions (see Table1) and answers were given in the form of numeric values ranging from 1 (I do not agree) to 5 (I do co agree).

\section{B. Data Acquisition}

Surface electromyography (EMG) signals were recorded from eight elbow and shoulder muscles: infraspinatus (IS), trapezius superior (TS), deltoid anterior (DA), deltoid medial (DM), pectoralis major (PM), biceps brachii (BB), triceps brachii long head (TBL) and brachioradialis (BRD). Electrodes were placed according to published guidelines [14]. Signals were acquired with the EMG100C system (Biopac Systems, Inc.) at a sampling rate of $1000 \mathrm{~Hz}$ and a gain of 500 using a pair of disposable $\mathrm{Ag} / \mathrm{AgCl}$ disc electrodes on each muscle $(1 \mathrm{~cm}$ in diameter, $1.5 \mathrm{~cm}$ interelectrode distance; EL501 foam electrode, Biopac Systems, Inc.). A notch filter was used to remove $50-\mathrm{Hz}$ interference.

\section{Data Analysis}

Resting periods were manually excluded from EMG signals. Resulting movement segments were concatenated and high-pass filtered using a zero-phase Butterworth $(n=6)$ filter, with a cutoff frequency of $50 \mathrm{~Hz}$. After de-meaning, linear envelopes were calculated and normalized to the maxima. The resulting 30 envelopes were then averaged for each movement to obtain the mean envelope. Signal length was normalized in time to 100 points. The control structure was extracted from the EMG of each subject's arm using the nonnegative matrix factorization algorithm. This represents the activity of the recorded muscles as a linear combination of time invariant muscle synergies, each activated by a timevarying activation coefficient. The model can be mathematically expressed as:

$$
\boldsymbol{D}(t)=\sum_{i=1}^{N} H_{i}(t) \cdot \boldsymbol{W}_{i}(t)+\boldsymbol{\varepsilon}
$$

where $\mathbf{D}(t)$ is the vector of EMG signals at time $t$, in the muscle space, $\mathrm{N}$ is the number of muscle synergies, $\mathbf{W}_{i}$ is the time-invariant muscle vector representing the $i$ th muscle synergy, $\mathrm{H}_{\mathrm{i}}(\mathrm{t})$ is the nonnegative time-varying activation coefficient for the $i$ th synergy and $\boldsymbol{\varepsilon}$ is any residual activity unexplained by linear combination. To set N, we successively increased the number of synergies extracted, from one to the number of muscles recorded, and selected the minimum number of synergies required for an EMG reconstruction Variance Accounted For (VAF) of $90 \%$.

\section{Inter-limb similarity and improvement measures}

The inter-limb similarity was defined as the similarity found between the synergies or activation coefficients of the right and left hand of the same subject when performing a given movement. The inter-limb similarity was quantified using the scalar product and the cross-correlation coefficient, for synergies and activation coefficients respectively. Scalar products were calculated after normalizing the vector norm of each synergy $\left(\mathbf{W}_{\mathrm{i}}\right)$ to one, while cross-correlation coefficients were calculated as the maximum values of the absolute crosscorrelations of the activation vectors $\left(\mathrm{H}_{\mathrm{i}}\right)$. We considered that the matching synergies of the left and right arm were the pair of synergies resulting in a higher scalar product. The activation coefficients were matched according to the matching of their corresponding synergies.

The similarity improvement was defined as the difference between the inter-limb similarity obtained with VF and the inter-limb similarity obtained without VF. Positive improvement values indicate an increase in the inter-limb similarity due to the VF and vice versa. Improvement measures were separately calculated for synergies and activation coefficients and therefore, they keep the units of the corresponding similarity measures in each case. We examined the impact of VF on the motor performance of individual muscles by calculating the absolute differences between the muscle-weights of the matching synergies of right and left arm. These measures were obtained with and without VF and subsequently compared between them. In order not to bias the results, we removed the subject for which VF did to improve inter-limb similarity.

Wilcoxon signed rank tests were used to assess differences between inter-limb similarities $(p<0.05)$. Friedman tests were used to assess differences across different subjects and muscles $(\mathrm{p}<0.05)$.

\section{RESULTS}

A control structure of two synergies (and corresponding activation coefficients) was sufficient to explain at least the $90 \%$ of the VAF variability of the 12 movements of almost all subjects. Certain subjects, though, needed three synergies to explain the $90 \%$ of the variability of some movements. However, because in these cases the VAF variability accounted by the two-synergy control structure did not go below $85 \%$, we used the two-synergy control structure in order to facilitate comparisons.

Fig. 1 shows the inter-limb similarity exhibited by each subject averaged across the 12 movements under analysis. In all cases, mean inter-limb similarity of the control structure was very high, ranging between 0.85 and 0.95 for synergies, and 0.95 and 0.98 for activation coefficients. The Friedman tests revealed that the intersubject differences in the interlimb similarity differences were significant in the case of the activation coefficients $(\mathrm{p}=0.0135)$, but not in the case of the synergies $(p=0.0845)$. However, this last result is likely to be due to the high variability found across movements in the inter-limb similarity of synergies.

Fig. 2 shows the improvement of the inter-limb similarity induced by the presence of VF. The mean improvement induced in synergies and activation vectors was of the order of $8.09 \pm 7.71 \%$ and $2.87 \pm 4.06 \%$ respectively. However, Wilcoxon Signed Rank test determined that the differences in 
the magnitude improvement induced by VF in synergies and activation vectors was statistically insignificant $(\mathrm{p}>0.05)$.
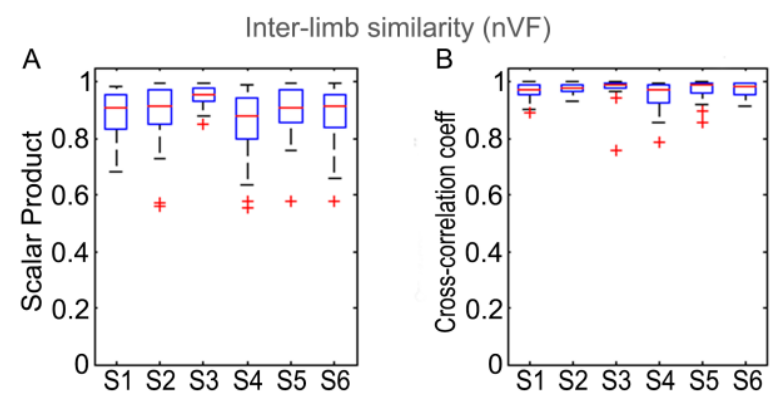

Figure 1 Inter-limb similarity of (A) synergies and (B) activation coefficients. Similarity measures are expressed as scalar products for synergies (A) and cross-correlation coefficients for activation coefficients (B). Each box represents the inter-limb similarity exhibited by a subject averaged across the 12 movements under analysis. The edges of the box indicate the $25^{\text {th }}$ and 75 th percentiles of the inter-limb similarity and the central red line indicates the median. The whiskers extend to the most extreme data point not considered outliers. Outliers are plotted as red crosses.

Almost all subjects experienced an improvement in their inter-limb similarity due to the VF. In particular, VF improved the inter-limb similarity of synergies in 5 subjects and the inter-limb similarity of activation coefficients in 4 subjects. Subject \#6 (S6) was the only subject that showed a decrease in the inter-limb similarity of synergies and activation vectors due to the VF. Interestingly, S6 was the only subject reporting that the VF disturbed him during the execution of movements (Table 1). Also, S6 was the only subject that had never practiced the exercises under study before.
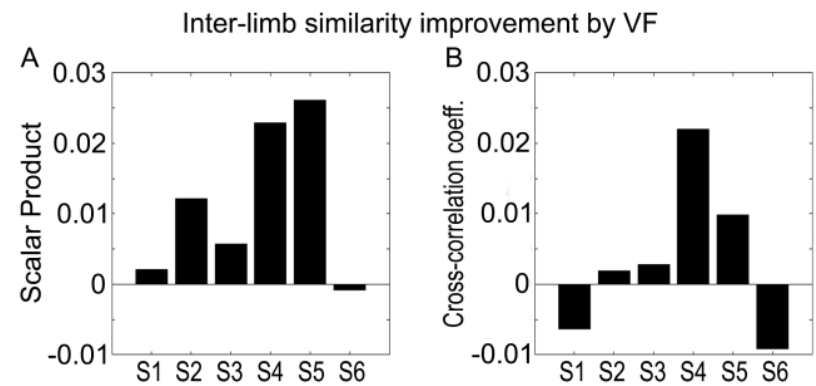

Figure 2 Improvement of the inter-limb similarity of (A) synergies and (B) activation coefficients due to the visual feedback. The improvement measure is computed as the difference of the inter-limb similarity shown with and without VF, in such a way that positive bars indicate a reduction in the interlimb similarity due to the presence of VF and vice versa. Bars are the mean improvement of each subject averaged across the 12 movements.

TABLE I.

AUTO-EVALUATION QUESTIONNAIRE

\begin{tabular}{|l|l|l|l|l|l|l|}
\hline \multirow{2}{*}{} & \multicolumn{7}{|c|}{ Subjects } \\
\cline { 2 - 7 } & \multicolumn{1}{|c|}{ S1 } & \multicolumn{1}{|c|}{ S2 } & \multicolumn{1}{c|}{ S3 } & \multicolumn{1}{c|}{ S4 } & \multicolumn{1}{c|}{ S5 } & \multicolumn{1}{c|}{ 66 } \\
\hline Q1 & 5 & 5 & 5 & 5 & 5 & 1 \\
\hline Q2 & 1 & 1 & 1 & 1 & 1 & 3 \\
\hline Q3 & 1 & 1 & 1 & 3 & 1 & 1 \\
\hline Q4 & 5 & 4 & 5 & 5 & 5 & 5 \\
\hline Q5 & 1 & 4 & 2 & 1 & 3 & 1 \\
\hline
\end{tabular}

Table I. Answers given by the subject in the auto-evaluation questionnaire delivered after the experiment. Q1 - I had practiced these exercises before. Q2 - The mirror made me lose the focus. Q3 - I sometimes forgot to look to the mirror. Q4 - I tried to do movement repetitions as similar as possible. Q5 - I found big differences between the executions of movements done with the right or left arm. Answers were given as numeric values ranging from 1 (I do not agree) to 5 (I fully agree).

Fig. 3 shows the inter-limb differences found in the muscle-weight of synergies with and without VF. The bars indicate that VF increases the inter-limb synergy similarity by reducing synergy-weight differences between right and left arm. This phenomenon was found in all muscles except in TS and PM. However, such differences might be insignificant since Friedman test revealed that the effect of VF does not depend on the muscle.

Fig. 4 shows two case examples of how the VF can affect the activation coefficient. The first example (Fig4A) depicts the most common effect of VF on the activation vectors, i.e., the cases where VF improves the inter-limb similarity. In these cases, although the inter-limb similarity found without $\mathrm{VF}$ is high, there is further scope for improving inter-limb similarity. Thus, VF tends to push the left activation coefficient towards the form of the right activation coefficient. In contrast, in some cases (Fig4A), the inter-limb similarity found without VF is so high that VF has little scope for increasing such similarity. This is the most common reason found case-by-case why the VF appears to reduce the inter-limb similarity of activation coefficients.

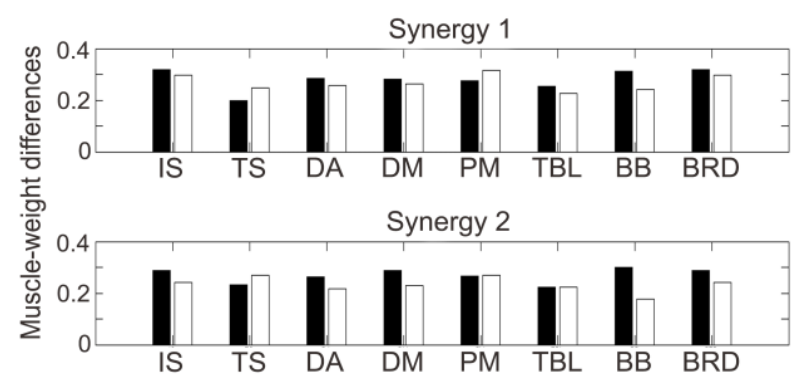

Figure 3 Changes induced by VF in the muscle-weights of synergies. Bars indicate absolute differences in the muscle-weights of synergies between left and right arm without VF (black bars) and with VF (white bars). Values are averaged across all subjects and movements. Muscles are infraspinatus (IS), trapezius superior (TS), anterior deltoid (DA), medial deltoid (DM), pectoralis major (PM), triceps brachii long head (TBL), biceps brachii (BB), and brachioradialis (BRD).
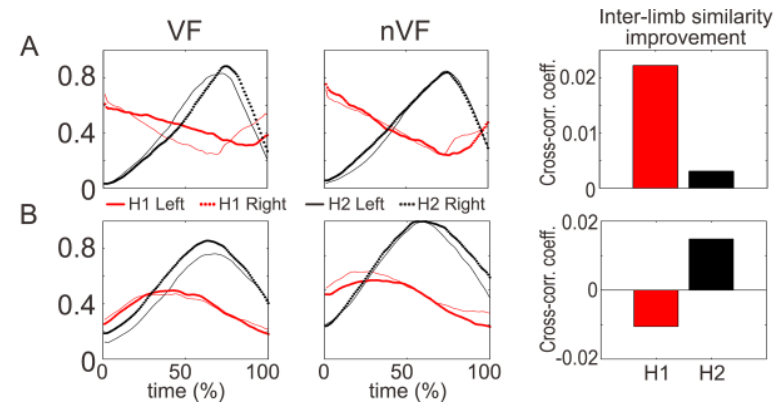

Figure 4 The effect of VF on activation coefficients: case examples. A Increase of inter-limb similarity due to VF. B - Decrease of inter-limb similarity due to VF. Dotted curves are right arm activation coefficients and full curves are left arm activation coefficients of synergy 1 (red) and synergy 2 (black). Bars indicate the inter-limb similarity improvement caused by VF in the activation coefficients of synergy 1 (red) and synergy 2 (black). The similarity improvement is expressed as the difference between the inter-limb similarities found with VF, minus the inter-limb similarity found without VF. Each case example corresponds to a representative subject (S3 and S4). 


\section{DISCUSSION}

This study analyzes the effectiveness of VF to modify the control structure of the upper-limb in a potential rehabilitation scenario. The major findings are as follows. (1) VF can effectively increase the inter-limb similarity of synergies and activation coefficients. (2) In the case of synergies, the improvement is achieved by reducing the interlimb muscle-weight differences of synergies (3). In the case of activation coefficients, the improvement is achieved by approximating the activation coefficient of the left arm towards the activation coefficient of the right arm. (4) The magnitude of improvement depends on subjects. Notably, the subject who reported confusion when using VF did not show any VF-driven similarity improvement. (5) Similarly, VF may not be effective in the cases where inter-limb similarity is almost total in the absence of VF.

Our results suggest that VF is able to effectively modify the control structure of the upper-limb towards a more optimized control structure. In particular, we show that VF brings the activation coefficients of the left arm closer to the activation vectors of the right arm and many studies demonstrate that the dominant hand exhibits advanced motor performance in terms of speed, precision and coordination $[15,16]$. Latash et al. suggested that the control structure might admit certain degree of variability, so that if a muscle introduces an error into the expected motor outcome, the other muscles may modify their contributions to compensate this error [17]. Therefore, it is presumable that VF, as part of the sensorimotor adaptation system, improves the motor performance of the upper-limb by correcting the control structure of movements during their execution. From the point of view of rehabilitation, these results are specially promising for two reasons. First, stroke-driven impaired coordination mainly arises from the alteration of the activation coefficients. Thus, VF may help modifying such activation coefficients to enhance motor performance. Second, it has been suggested that the control of the paretic side is similar to the control of a healthy nondominant side [18]. Thus, it is presumable that these results can be translated to the hemiparesis scenario found in stroke.

It has to be noted that the magnitude of the effect of VF shows a significant inter-subject dependence. It seems that $\mathrm{VF}$ is ineffective in subjects finding VF disturbing. Therefore, it is expected that patients manifesting cognitive problems may not benefit from the use of VF. The individual basal inter-limb similarity might also explain such differences. Apparently, VF has no effect (or even a subtle negative effect) in the cases in which, inter-limb similarity is very high in absence of VF. Zanone reported that VF has a greater impact on motor performance in children who have not yet achieved motor proficiency [19]. Thus, VF might have a maximum performance limit from which, the scope to motor improvement is too little as to produce any substantial effect in the inter-limb similarity. Fortunately, this is not the case of a rehabilitation scenario in which the motor differences between the affected and non-affected side are obvious. Be as it may be, all these considerations should be taken into account to design effective rehabilitations strategies based on VF. For this reason, we are currently working to apply this protocol to patients with stroke to study how VF affects the rehabilitation process. Indeed, further research is needed to identify the best ways to exploit the potential of VF in rehabilitation.

\section{REFERENCES}

[1] American Heart Association (AHA), "Heart Disease and Stroke Statistics: 2014 Update," Circulation, vol. 139, no. 3, 2014.

[2] T. Flash and B. Hochner, "Motor primitives in vertebrates and invertebrates," Curr Opin Neurobiol, vol. 15, pp. 660-66, 2005.

[3] E. Bizzi, V. C. K. Cheung, A. d'Avella, P. Saltiel and M. C. Tresch, "Combining modules for movements," Brain Res Brain Res Rev, vol. 57, pp. 125-33, 2008.

[4] V.C.K. Cheung, L. Piron, M. Agostini et al. "Stability of muscle synergies for voluntary actions after cortical stroke in humans," PNAS, vol. 106, no. 46, 19563-68, 2009.

[5] J. Roh, W.Z.Rymer, E.J. Perreault et al., "Alterations in upper limb muscle synergy structure in chronic stroke survivors," J Neurophysiol, vol. 109, no. 3, 2013

[6] V.C.K. Cheung, A. Turolla, M. Agostini et al., "Muscle synergy patterns as physiological markers of motor cortical damage," PNAS, vol. 109, no. 36, pp. 14652-14656, 2012.

[7] O. Urra, A. Casals, and R. Jané, "Study of Synergy Patterns during the Execution of Stroke Rehabilitation Exercises", in Proc. $32^{\text {nd }}$ Congreso Annual de la Sociedad Española de Ingeniería Biomédica, Barcelona, 2014.

[8] H. Thieme, J. Mehrholz, M. Pohl, J. Behrens and C. Dohle, "Mirror therapy for improving motor function after stroke", Cochrane Database Syst Rev, vol. 3, 2012.

[9] G. Yavuzer, R. Selles, R. Sezer, N. Sütbeyaz, S. Bussmann et al., "Mirror therapy improves hand function in subacute stroke: a randomized controlled trial," Arch Phys Med Rehabil, vol. 89, pp. 39398, 2008.

[10] C. Dohle, J. Püllen, A. Nakaten. J. Küst, C. Rietz and H. Karbe, "Mirror therapy promotes recovery from severe hemiparesis: a randomized controlled trial," Neurorehabil Neural Repair, vol. 23, vol. 209-217, 2009.

[11] M. Garry, A. Loftus and J. Summers, "Mirror, mirror on the wall viewing a mirror reflection of unilateral hand movements facilitates ipsilateral M1 excitability," Exp Brain Res, vol. 163, pp. 118-122, 2005.

[12] I. Nojima, T. Mima, S. Koganemaru, M. N. Thabit, H. Fukuyama and T. Kawamata, "Human motor plasticity induced by mirror visual feedback," J Neurosci, vol. 32, pp. 1293-1300, 2012.

[13] O. Urra, A. Casals, and R. Jané, "Synergy Analysis as a Tool to Design and Assess an Effective Stroke Rehabilitation," in Proc. 35th Annual International Conference of the IEEE Engineering in Medicine and Biology Society (EMBC), Chicago, 2014.

[14] A.O. Perotto. Anatomical guide for the electromyographer; the limbs and trunk. Sprigfield, IL: C. C. Thomas, 2005.

[15] P.E.G. Bestelmeyer and D. Carey, "Processing bias towards the preferred hand: valid and invalid cueing of left-versus right-hand movements, “ Neuropsychologia, vol. 42, pp. 1162-67, 2004.

[16] K. Kauranen K and H. Vanharanta, "Influence of age, gender, and handedness on motor performance of upper and lower extremities," Percept Mot Skills, vol. 82, pp. 512-25, 1996.

[17] M.L. Latash, J.F. Scholz, F. Danion F and G. Schoånner G. "Structure of motor variability in marginally redundant multifinger force production tasks", Exp Brain Res, vol. 141, pp. 153-165, 2001.

[18] J. Chiang, J. Wang and M.J. McKeown. "Study of stroke condition and hand dominance using a Hidden Markov, Multivariate Autoregressice (HMM-mAR) Network Framework", in Proc 30th Annu International IEEE EMBS Conf, Vancouver, 2008.

[19] P.G. Zanone. "Tracking with and without target in 6- to 15-year-old boys," J Mot Behav, vol. 22, no. 2, pp. 225-49, 1990. 